\title{
Ampliação da distribuição de Molossops neglectus Williams \& Genoways (Chiroptera, Molossidae) para o Sul da América do Sul ${ }^{1}$
}

\author{
Itiberê P. Bernardi 2; Atenisi Pulchério-Leite ${ }^{2,3}$; João M. D. Miranda ${ }^{2,3}$ \& Fernando C. Passos ${ }^{2}$ \\ ${ }^{1}$ Contribuição número 1700 do Departamento de Zoologia, Universidade Federal do Paraná. \\ 2 Laboratório de Biodiversidade, Conservação e Ecologia de Animais Silvestres, Departamento de Zoologia, Universidade \\ Federal do Paraná. Caixa Postal 19020, 81531-980 Curitiba, Paraná, Brasil. \\ E-mail: sturnira@gmail.com; atenasbat@terra.com.br; guaribajoao@yahoo.com.br; fpassos@ufpr.br \\ ${ }^{3}$ Bolsista do CNPq.
}

\begin{abstract}
Range extension of Molossops neglectus Williams \& Genoways (Chiroptera, Molossidae) in Southern Brazil. The present study reports on the first record Molossops neglectus Williams \& Genoways, 1980 in the State of Rio Grande do Sul and provides the southernmost limit of the distribution of this species in South America. Three specimens were collected in Frederico Westphalen $\left(27^{\circ} 21^{\prime} 33^{\prime \prime} \mathrm{S}\right.$ and $\left.53^{\circ} 23^{\prime} 40^{\prime \prime} \mathrm{W}\right)$ within the domain of the Deciduous Seasonal Forest. Morphometric and taxonomically diagnostic characters are provided for the collected specimens, as well as relevant data to the understanding of the reproductive biology of the species. An ectoparasitic hemipteran is also reported.
\end{abstract}

KEY WORDS. Mammalia; Rio Grande do Sul; Seasonal Deciduous Forest.

RESUMO. É reportado o primeiro registro de Molossops neglectus Williams \& Genoways, 1980 para o Estado do Rio Grande do Sul e se estabelece um novo limite sul na distribuição da espécie na América do Sul. Foram

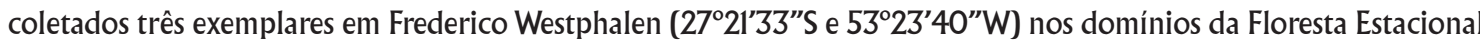
Decidual. Dos espécimes coletados, são apresentados dados morfométricos, diagnose e dados que contribuem para o conhecimento da biologia reprodutiva da espécie. É ainda citado um hemíptero ectoparasito.

PALAVRAS-CHAVE. Mammalia; Floresta Estacional Decidual; Rio Grande do Sul.

O gênero Molossops Peters, 1865, é composto atualmente por quatro espécies (SIMMONs 2005), das quais, três ocorrem em território brasileiro, Molossops (Molossops) temminckii (Burmeister, 1854), M. (Molossops) neglectus Willians \& Genoways, 1980 e M. (Neoplatymops) mattogrossensis (Vieira, 1942) (Gregorin \& TADDEI 2002, Simmons 2005, Peracchi et al. 2006). Inicialmente a espécie possuia registros conhecidos para o Suriname, norte do Brasil e norte do Peru (Ascorra et al. 1991). ОсHOA et al. (1993) reportaram sua ocorrência no leste da Venezuela. Posteriormente $M$. neglectus foi registrada ocorrendo na Guyana e no sul da Colômbia (Lim \& Engstrom 2001) e na Argentina (BARquez et al. 1993, 1999). Recentemente, GREGORIN et al. (2004) ampliaram a distribuição da espécie registrando-a em quatro localidades na região sudeste do Brasil. Neste trabalho, é reportado o novo limite sul de distribuição para M. neglectus, e fornecidos alguns dados biológicos que contribuem substancialmente para o escasso conhecimento da biologia da espécie.

O município de Frederico Westphalen, no Rio Grande do Sul, está localizado na região do Médio Alto Uruguai, nos do- mínios da Floresta Estacional Decidual, sob as coordenadas $27^{\circ} 21^{\prime} 33^{\prime \prime}$ S e $53^{\circ} 23^{\prime} 40^{\prime \prime} \mathrm{W}$, a uma altitude de $522 \mathrm{~m}$. O clima da região é do tipo Cfa segundo Köppen. A temperatura média anual é em torno $18^{\circ} \mathrm{C}$, com máximas no verão podendo atingir $41^{\circ} \mathrm{C}$ e mínimas no inverno atingindo valores inferiores a $0^{\circ} \mathrm{C}$. A precipitação média anual é elevada, geralmente entre 1.800 e $2.100 \mathrm{~mm}$ bem distribuídos ao longo do ano. As capturas foram realizadas em um fragmento florestal de aproximadamente 35 hectares, distante três quilômetros da zona urbana do município, na área do Parque Municipal de Turismo e Lazer da Faguense.

Para as capturas foram utilizadas seis redes de neblina "mist nets" (7 x 2,5 m) posicionadas em trilhas, clareiras, borda de mata, bem como perpendicularmente a cursos d'água, a uma altura de $0,5 \mathrm{~m}$ do solo. Os exemplares coletados como material testemunho foram fixados de acordo com Vizotтo \& TADDEI (1973) e posteriormente tombados na coleção científica do Departamento de Zoologia, UFPR, Sessão de Mastozoologia, sob os números CCMZ/UFPR 220, CCMZ/UFPR 275 e CCMZ/

Revista Brasileira de Zoologia 24 (2): 505-507, junho 2007 
UFPR 274. Os espécimes foram mensurados de acordo com os critérios descritos em TADdeI et al. (1998). As abreviações das medidas externas e cranianas foram as seguintes: An: comprimento do antebraço; Ccr: comprimento total do crânio; Ci: comprimento côndilo-incisivo; Lcx: largura da caixa craniana; Lm: largura através dos molares superiores; C-M: comprimento da série de dentes superiores; Cm: comprimento da mandíbula. Ressalte-se que, conforme os critérios apresentados por TADDEI et al. (1998), o comprimento da mandíbula e o comprimento total do crânio foram tomados de modo a não estender a medida à frente dos incisivos, mas sim, até a região mais anterior destes.

Foram coletados três indivíduos de M. neglectus. Uma fêmea adulta (CCMZ/UFPR 220) em 01-X-2005 às 05h40min; uma fêmea adulta (CCMZ/UFPR 275) em 05-I-2006 às 21h20min apresentando sinais de lactação e um macho adulto (CCMZ/UFPR 274) em 05-I-2006 às 22h20min com os testículos na bolsa escrotal. A fêmea (CCMZ/UFPR 275) apresentou um ectoparasito do gênero Hesperoctenes Kirkaldy, 1906 (Hemiptera, Polyctenidae) (G. Graciolli, Universidade Federal de Mato Grosso do Sul, comunicação pessoal).

Descrição dos exemplares coletados (ver também Lim \& Engstrom 2001, Gregorin \& TAdDei 2002, Gregorin et al. 2004):

Os espécimes coletados apresentaram lábio superior liso, pontos de origem das margens internas das orelhas sobre a cabeça, separados por um espaço superior a $4,5 \mathrm{~mm}$, antitrago voltado para trás e sem constrição acentuada, crista posterior do terceiro molar tão longa quanto a primeira e incisivos $1 / 1$, situando os exemplares no gênero Molossops de acordo com BARQUEz et al. (1999) e GREGORIN \& TADDEI (2002). A identificação dos exemplares em nível específico seguiu BARQuez et al. (1999) e Gregorin \& TADDEI (2002) onde foram observados, a ausência de granulações conspí-

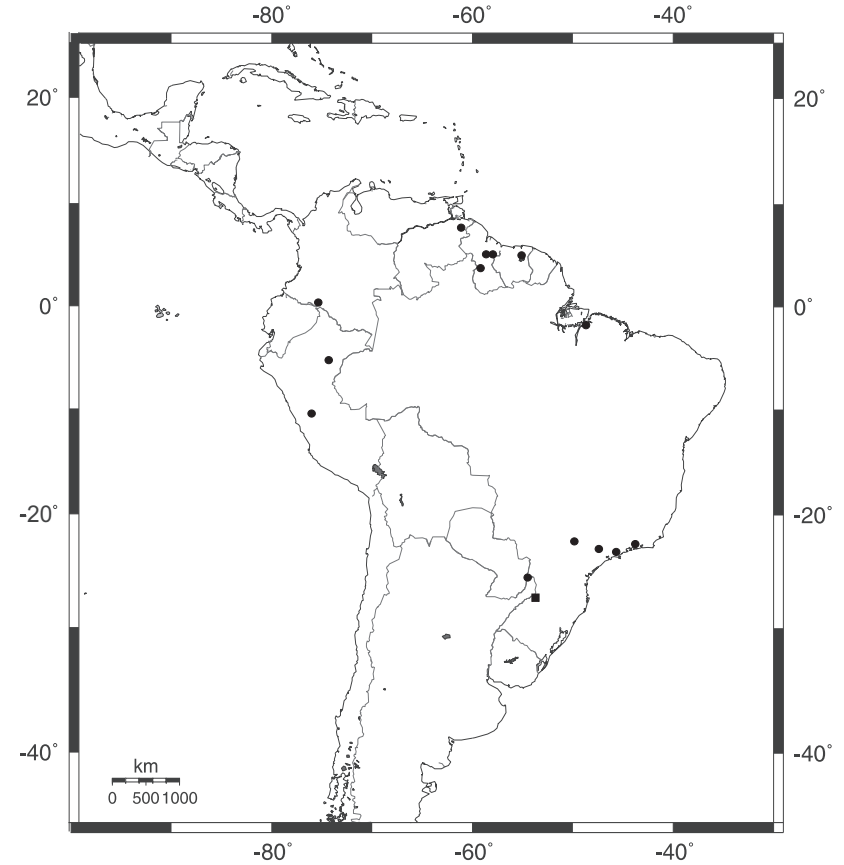

Fig. 1. Distribuição de Molossops neglectus. Localidades previamente conhecidas da ocorrência $(\bullet)$; Frederico Westphalen, Rio Grande do Sul $\left(27^{\circ} 21^{\prime} \mathrm{S}\right.$ e $\left.53^{\circ} 23^{\prime} \mathrm{W}\right)$, localidade aqui reportada (匹).

cuas na superfície dorsal do antebraço, a pelagem dorsal marrom escuro com as bases distintamente brancas, o comprimento total do crânio variando entre 15,5 e $16,5 \mathrm{~mm}$ e o comprimento do antebraço entre 37,1 e $38,1 \mathrm{~mm}$.

Tabela I. Medidas (mm) obtidas de Molossops neglectus provenientes do Rio Grande do Sul, Brasil (espécimes 7, 8 e 9). Os dados dos outros exemplares foram obtidos em GREGORIN et al. (2004).

\begin{tabular}{ccccccccc}
\hline Espécimes & Sexo & $\mathrm{Na}$ & $\mathrm{Ccr}$ & $\mathrm{Ci}$ & $\mathrm{Lcx}$ & $\mathrm{Lm}$ & $\mathrm{C}-\mathrm{M}$ & $\mathrm{Cm}$ \\
\hline 1 & 1 Fêmea & 37,1 & 15,5 & 15,3 & 8,4 & 7,1 & 6,2 & 10,5 \\
2 & 1 Fêmea & 38,1 & 15,7 & 15,4 & 8,1 & 7,3 & 6,2 & 10,6 \\
3 & 1 Macho & 37,1 & 16,5 & 16,2 & 8,3 & 7,6 & 6,6 & 11,3 \\
4 & 1 Macho & 36,7 & 15,8 & 14,3 & 7,8 & 6,4 & 5,6 & 11,2 \\
5 & 1 Macho & 36,9 & 16,4 & 15,6 & - & 7,1 & 6,1 & - \\
6 & 1 Macho & 36,6 & 16,6 & - & 8,2 & 7,4 & 5,9 & 12,7 \\
7 & 1 Fêmea & 36,0 & 15,5 & 14,8 & 8,2 & 7,2 & 5,9 & 12,0 \\
8 & 1 Fêmea & 35,1 & 17,1 & 15,4 & 8,8 & 7,7 & 6,3 & 12,2 \\
9 & 3 Machos & $38,0-40,0$ & $18,6-19,8$ & $17,0-18,2$ & $8,4-8,6$ & $7,9-8,4$ & $6,9-7,3$ & $12,5-13,2$ \\
9 & 7 Fêmeas & $36,0-39,0$ & $16,7-17,9$ & $15,7-16,8$ & $8,0-8,4$ & $7,5-8,0$ & $6,4-6,8$ & $11,5-12,5$ \\
\hline
\end{tabular}

1) CCMZ/UFPR 220, 2) CCMZ/UFPR 275 e 3) CCMZ/UFPR 274 provenientes do Rio Grande do Sul, Frederico Westphalen (27² $21^{\prime} 33^{\prime \prime} S$

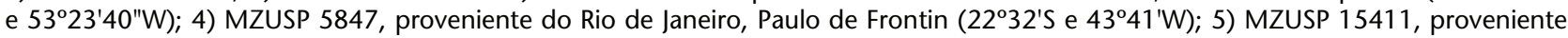

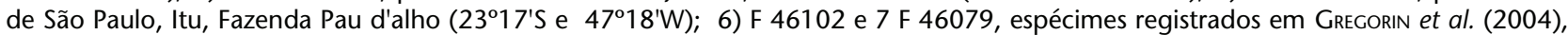
provenientes de São Paulo, Estação Ecológica de Caetetus (22 $23^{\prime}$ 'S e $\left.49^{\circ} 40^{\prime} \mathrm{W}\right)$, tombados na coleção científica do Departamento de Apoio, Produção e Saúde Animal, UNESP/Araçatuba; 8 holótipo descrito em WiLlaAms \& Genoways (1980), do Suriname, Powaka ( $5^{\circ} 27^{\prime} N$ e $\left.55^{\circ} 5^{\prime} \mathrm{W}\right) ; 9$ espécimes registrados em LIM \& ENGSTROM (2001), provenientes de três locais na Guyana, Arampa $\left(5^{\circ} 31^{\prime} \mathrm{N}\right.$ e $\left.58^{\circ} 18^{\prime} \mathrm{W}\right)$, Kamakabra $\left(5^{\circ} 28^{\prime} \mathrm{N}\right.$ e $\left.58^{\circ} 16^{\prime} \mathrm{W}\right)$ e Iwokrama Forest $\left(4^{\circ} 22^{\prime} \mathrm{N}\right.$ e $\left.58^{\circ} 51^{\prime} \mathrm{W}\right)$. 
As medidas morfométricas (Tab. I) dos exemplares procedentes de Frederico Westphalen, Rio Grande do Sul, mostram-se congruentes com o encontrado em outros pontos da distribuição da espécie, e, somadas aos demais caracteres apresentados, permitiram a identificação segura dos espécimes como M. neglectus e não M. temminckii, espécie congênere já registrada no Rio Grande do Sul (Silva 1994).

Os dados aqui reportados caracterizam o primeiro registro de M. neglectus para o Estado do Rio Grande do Sul, bem como, estabelecem um novo limite sul para a distribuição da espécie na América do Sul, cerca de $250 \mathrm{~km}$ ao sul do limite até então conhecido, no Parque Nacional Iguazu, Província de Misiones, Argentina (BARquez et al. 1993, 1999, BARQuez 2006, Gregorin et al. 2004) (Fig. 1). Embora a ocorrência de M. neglectus fosse esperada para o Paraná (Sekiama et al. 2001) e para Santa Catarina (Cherem et al. 2004), os dados aqui apresentados são os únicos registros da ocorrência da espécie para a região sul-brasileira.

\section{AGRADECIMENTOS}

A Gustavo Graciolli, pela identificação do ectoparasita; Marcio R. Pie pela tradução do resumo; Nathalia Y.K. Oliveira pela confecção das fotografias; Lucas M. Aguiar pela confecção do mapa.; Eduardo Grotto, Jonas Sponchiado e Suelen H. Roani pelo auxílio em campo e Rafael Ferigollo, pelo apoio logístico.

\section{REFERÊNCIAS BIBLIOGRÁFICAS}

Ascorra, C.F.; D.E. Wilson \& C.O. Handley. 1991. Geographic distribution of Molossops neglectus Williams and Genoways (Chiroptera: Molossidae). Journal of Mammalogy 74: 828-830.

BARQUEZ, R.M. 2006. Orden Chiroptera, p 56-86. In: R.M. BARQUEZ; M.M. Díaz \& R.A. OJeda (Eds). Mamíferos de Argentina, sistemática y distribución. Tucumán, SAREM, 359p.

Barquez, R.M., N.P. Giannini \& M.A. Mares. 1993. Guide to the bats of Argentina (guia de los murcielagos de Argentina). Norman, Special Publications of Oklahoma Museum of Natural History, 119p.

Barquez, R.M.; M.A. Mares \& J.K. Braun. 1999. The Bats of Argentina. Special Publications of the Museum of Texas Tech University 42: 1-275.

Cherem J.J.; P.C. Simões-Lopes; S. Althoff \& M.P. Graipel. 2004.
Lista dos Mamíferos do Estado de Santa Catarina, Sul do Brasil. Mastozoología Neotropical 11 (2): 151-184.

Gregorin R. \& V.A. TAdDei. 2002. Chave artificial para a identificação de Molossídeos brasileiros (Mammalia, Chiroptera). Mastozoología Neotropical 9 (1): 13-32.

Gregorin, R.; B.K. Lim; W.A. Pedro; F.C. PAssos \& V.A. Taddei. 2004. Distributional extension of Molossops neglectus (Chiroptera, Molossidae) into southeastern Brazil. Mammalia 68: 233237.

Lim, B.K. \& M.D. Engstrom 2001. Species diversity of bats (Mammalia: Chiroptera) in Iwokrama Forest, Guyana, and the Guianan subregion: implications for conservation. Biodiversity and Conservation 10: 613-657.

Ochoa, G.J., P.J. Soriano, D. Lew \& M.C. OJeda. 1993. Taxonomic and distributional notes on some bats and rodents from Venezuela. Mammalia 57: 393-400.

Peracchi, A.L.; I.P. Lima; N.R. Reis; M.R. Nogueira \& H.O. Filho. 2006. Ordem Chiroptera, p. 155-234. In: N.R. ReIs; AL. Peracchi; W.A. Pedro \& I.P. Lima (Eds). Mamíferos do Brasil. Curitiba, Governo do Paraná, SEMA, SBZ, 437p.

Sekiama, M.L.; N.R. Reis; A.L. Peracchi \& V.J. Rocha. 2001. Morcegos do Parque Nacional do Iguaçu, Paraná (Chiroptera, Mammalia). Revista Brasileira de Zoologia 18: 749-754

SILVA, F. 1994. Mamíferos silvestres: Rio Grande do Sul. Porto Alegre, Fundação Zoobotânica do Rio Grande do Sul, 246p.

Simmons, N.B. 2005. Order Chiroptera, p. 137-241. In: D.E. WILSON \& D.M. REEDER. (Eds). Mammals species of the world: a taxonomic and geographic reference. Washington, Smithsonian Institution Press, vol. 1, 739p.

Taddei, V.A.; C.A. Nobile \& E. Morielle-Versute. 1998. Distribuição geográfica e análise morfométrica comparativa em Artibeus obscurus (Schinz, 1821) e Artibeus fimbriatus Gray, 1838 (Mammalia, Chiroptera, Phyllostomidae). Ensaios e Ciência 2 (2), CD-ROOM.

VizotTo, L.D. \& V.A. TAdDEI. 1973. Chave para determinação de quirópteros brasileiros. Boletim de Ciências, Faculdade de Filosofia, Ciências e Letras 1: 1-72.

Williams, S.L. \& H.H. Genoways. 1980. Results of the Alcoa Foundation-Suriname Expeditions. IV. A new species of bat of the genus Molossops (Mammalia: Molossidae). Annals of Carnegie Museum 49: 487-498.

Recebido em 25.X.2006; aceito em 14.V.2007. 